\title{
Role of preoperative MRI in guiding the surgical treatment decision in patients with invasive breast cancer
}

\author{
Atallah D, Moubarak M, Arab W, El Kassis N, Chahine G, Ghossain M, Salem C \\ Hôtel-Dieu de France University Hospital, Saint Joseph University, Beirut, Lebanon
}

\section{AIM}

To illustrate the importance of preoperative MRI in the surgical treatment decision in patients with invasive breast cancer

\section{METHODS}

We reviewed retrospectively the preoperative MRI (3 Tesla) of 169 patients operated at Hôtel-Dieu de France for invasive breast cancer. The sequences of 85 patients who underwent a radical treatment (mastectomy) were compared to those of 84 patients who benefited from conservative surgery. We estimated the tumor volume (TV) and breast volume (BV) on enhanced MRI and then we compared the tumor volume to breast volume ratio (TV/BV) in both groups.

\section{RESULTS}

- When compared to pathologic reports, the MRI seemed to slightly increase the estimation of tumor size, with a correction of the difference using the following formula: tumor size on pathology $(\mathrm{mm})=$ $(0.895$ * tumor size on MRI) + $1.101(\mathrm{~mm})$.

- The mean of tumor to breast volume ratio was $9,5 \%$ in the mastectomy group vs. $1.7 \%$ in the conservative treatment group ( $p$ $=0.000$ ).
- Volume ratio less than $4 \%$ seemed to favor the adoption of the conservative option in the surgical treatment decision in patients with invasive breast cancer ( $p$ $=0.000$ ).

- MRI had also helped the assessment of multifocality and multicentricity of tumor which were noted respectively in $57.4 \%$ and $54.4 \% \%$ of cases treated with mastectomy vs. $15.5 \%$ and $3.6 \%$ of cases treated conservatively $(p=0.000)$.

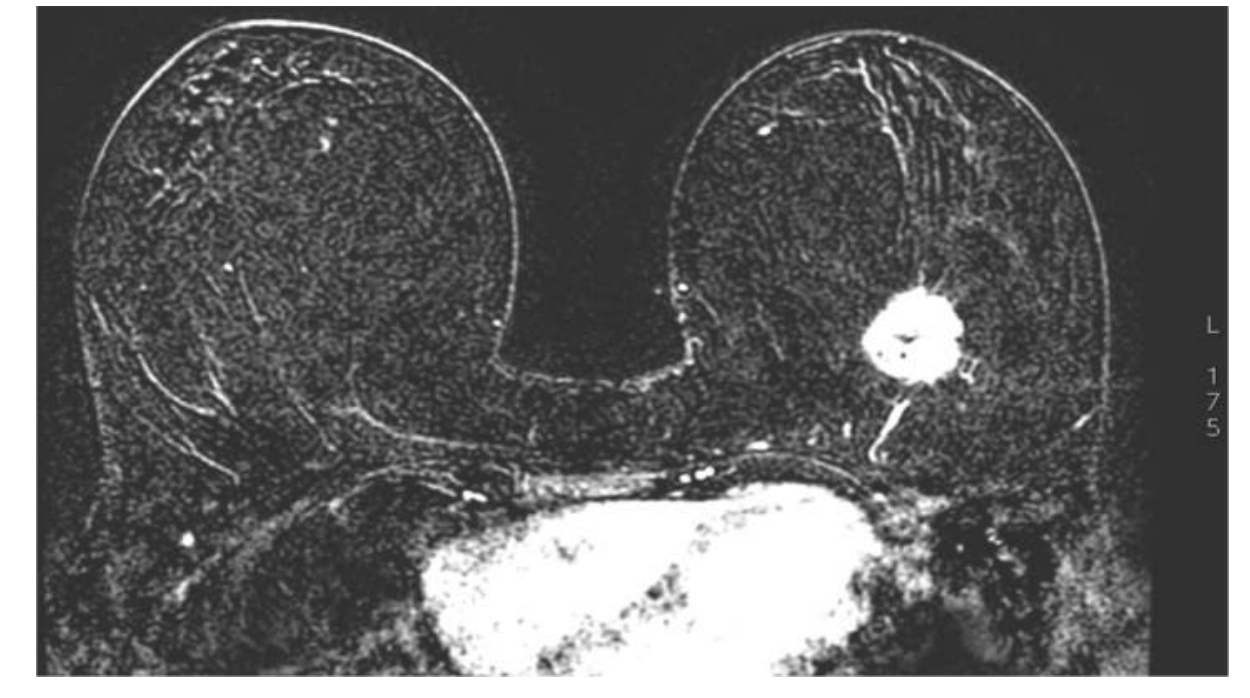

FIGURE I. Contrast-enhanced breast MR imaging (with subtraction post-pre) showing a high signal lesion
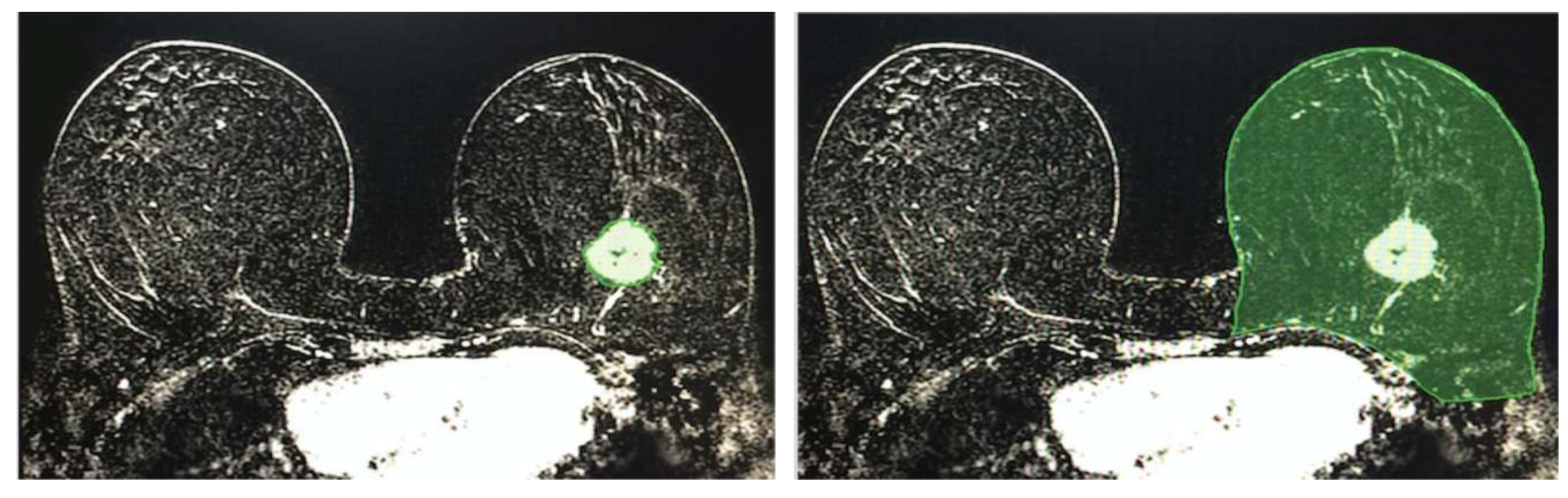

FIGURE 2 \& 3. Tumor-to-breast volume ratio (TBR) measured using a volume viewer on a contrast-enhanced sequence with subtraction

\section{CONCLUSION}

Our data suggest that preoperative MRI can aid the surgical treatment decision in patients with invasive breast cancer by assessing the tumor to breast volume ratio as well as by providing a full mapping of multifocal and multicentric lesions in the breast tissue. 